\title{
Family Aggregation of High Myopia: Estimation of the Sibling Recurrence Risk Ratio
}

\author{
Jane E. Farbrother, ${ }^{1}$ George Kirov, ${ }^{2}$ Michael J. Owen, ${ }^{2}$ and Jeremy A. Guggenheim ${ }^{1}$
}

Purpose. To estimate the sibling recurrence risk $\left(K_{\mathrm{S}}\right)$ and the sibling recurrence risk ratio $\left(\lambda_{\mathrm{S}}\right)$ for high myopia in a cohort in the United Kingdom.

MeTHOD. The recurrence risks for myopia and high myopia were estimated in the siblings of 296 randomly selected high myopes ascertained from an optometric practice population. A model using an age of onset of spectacle wear for myopia of $9.1 \pm 0.7$ years or younger was developed as a surrogate for high myopia. The influence of parental myopia on the sibling recurrence risk for high myopia was also evaluated.

Results. $K_{\mathrm{S}}$ was estimated ( $95 \%$ confidence limits) to be $10.0 \%$ $(5.9,14.8)$ and $\lambda_{\mathrm{s}}$ to be $4.9(2.8,7.6)$. High myopes without myopic parents were surprisingly common $(\sim 40 \%)$ and were less likely to have highly myopic siblings $\left(K_{\mathrm{S}} \sim 6 \%\right)$ than those with at least one myopic parent $\left(K_{\mathrm{S}} \sim 14 \%\right)$.

Conclusions. The sibling recurrence risk ratio reported herein $\left(\lambda_{\mathrm{S}} \sim 4.9\right)$ implies that the high penetrance autosomal dominant loci for high myopia identified to date account for only a minority of cases of high myopia in the United Kingdom. Furthermore, high-penetrance autosomal dominant inheritance or even high-penetrance recessive inheritance, per se, cannot account for most cases of high myopia. Instead, it may be necessary to consider high myopia as a "complex disease" resulting from the influence of either alleles of reduced penetrance ("susceptibility genes"), environmental factors, or both. (Invest Ophthalmol Vis Sci. 2004;45:2873-2878) DOI: 10.1167/iovs.03-1155

M yopia is a common ocular condition. ${ }^{1}$ In its extreme form, high myopia (usually defined as a refractive error $\leq$ $-6.00 \mathrm{D}$ ) forms the negative tail of the refractive error distribution and constitutes approximately $1 \%$ to $2 \%$ of the adult white population. ${ }^{2}$ Historically, high myopia has been regarded as an etiologically discrete entity from low myopia, ${ }^{3-5}$ albeit with a variety of classification thresholds. This theory seems to have been borne out by genetic linkage studies. ${ }^{6-9}$ The autosomal loci that have been mapped with certainty to

From the ${ }^{1}$ School of Optometry and Vision Sciences, Cardiff University, Cardiff, Wales, United Kingdom; and the ${ }^{2}$ Department of Psychological Medicine, University of Wales College of Medicine, Cardiff, Wales, United Kingdom.

Supported by National Eye Research Centre Grant SCIAD 015.

Submitted for publication October 21, 2003; revised April 13, 2004; accepted May 10, 2004

Disclosure: J.E. Farbrother, None; G. Kirov, None; M.J. Owen, None; J.A. Guggenheim, None

The publication costs of this article were defrayed in part by page charge payment. This article must therefore be marked "advertisement" in accordance with 18 U.S.C. $\$ 1734$ solely to indicate this fact.

Corresponding author: Jeremy A. Guggenheim, School of Optometry and Vision Sciences, Cardiff University, Redwood Building, King Edward VII Avenue, Cardiff CF10 3NB, UK;

guggenheim@cardiff.ac.uk. date constitute highly penetrant, dominant loci. ${ }^{6-8}$ Ascertainment of pedigrees with multiple affected individuals usually favor the detection of dominant over recessive loci, ${ }^{10}$ and hence the contribution of the highly penetrant dominant loci that have been identified to the overall prevalence of high myopia is unknown.

The success of a particular ascertainment scheme used for the molecular genetic study of a given condition depends on the number of contributory genetic loci, the magnitudes of their effects, their modes of inheritance, and their interaction with each other, with alleles at additional loci, and with environmental factors. Genetic epidemiologic investigations provide valuable insight into these questions. In particular, the power of molecular genetic studies to detect linkage can be estimated from the sibling recurrence risk $\left(K_{\mathrm{S}}\right)$ and its ratio to the population prevalence $\left(\lambda_{\mathrm{S}}\right),{ }^{11-13}$ as long as the potential contribution from familially aggregated environmental risk factors is not overlooked. ${ }^{14,15}$ In this study, estimates of these parameters were made using a randomly selected sample of high myopes identified from a British optometric practice population. These results have been presented in abstract form (Farbrother JE, et al. IOVS 2002;43:ARVO E-Abstract 1847).

\section{Subjects ANd Methods}

Ethical approval for these studies was granted by the Human Science Ethical Committee, Cardiff University. The research adhered to the tenets of the Declaration of Helsinki.

The recurrence risks for myopia and high myopia were estimated among the siblings of high myopes attending a group of 19 optometric practices in the north of England. The records for all patient attendances to the 19 practices between January 2000 and December 2001 were surveyed, with information obtained only from the most recently recorded visit for patients attending more than once. From the resultant set of 90,884 individual patient records, 1,846 subjects met our predefined criteria for high myopia (a spectacle refraction of $\leq-6.00$ $\mathrm{D}$ in the least minus meridian of each eye). The high myopia prevalence estimate (95\% confidence limits) in this population was thus $2.03 \%(1.94,2.12)$. Because the population from which these high myopes were ascertained almost certainly overrepresented subjects with visually significant refractive errors, there is a potential for upward bias in this population prevalence estimate. However, in the absence of a reliable estimate of high myopia prevalence in the United Kingdom or elsewhere in Europe, this estimate was used to calculate the sibling recurrence risk ratio $\left(\lambda_{\mathrm{s}}\right)$, since it was in agreement with an unbiased estimate of the prevalence of high myopia for whites in the United States. ${ }^{2}$ A randomly selected sample of 527 of the 1846 high myopes were contacted (Table 1). The principal method of contact was by mail. However, in an attempt to evaluate any potential response bias from subjects with a positive family history, ${ }^{16}$ questionnaires were also administered by telephone for a subset of the high myopes, with the method of contact being selected on the basis of whether a subject's home telephone number was listed in the telephone directory. Postal questionnaires were sent to 361 of the high myopes, along 
TABLE 1. Ascertainment Scheme

\begin{tabular}{lccc}
\hline & Mail & Telephone & Total \\
\hline Highly myopic probands contacted & 361 & 166 & 527 \\
Respondents & 161 & 135 & 296 \\
Included respondents* & 157 & 132 & 289 \\
Siblings of included respondents & 270 & 223 & 493 \\
Siblings with spectacle-wearing status known & 258 & 216 & 474 \\
Siblings wearing spectacles full time for myopia (FTSW) & 130 & 81 & 211 \\
Siblings with age of onset of myopia known & 122 & 59 & 181 \\
\hline
\end{tabular}

* Included respondents were those remaining after removal of families containing any siblings aged $\leq 15$ years and not wearing spectacles. Data are the number in each category.

with a covering letter encouraging return of the questionnaire even if the family history was negative. One of four researchers contacted the remaining 166 subjects using a standardized telephone questionnaire. In the case of both postal and telephone questionnaires, questions were designed to elicit information regarding premature birth, general and ocular health, age of onset of spectacle wear and number, age, gender, and spectacle-wearing status of siblings. ${ }^{17}$ If siblings were reported as myopes wearing spectacles full time, subjects were asked to indicate the age at which they began wearing spectacles. Subjects were also asked whether their parents wore either a full or part-time myopic refractive correction. The single ascertainment strategy used meant that multiplex families were more likely to be included in the study, because of chance ascertainment of any one affected person as a proband; however, our method of calculating $K_{\mathrm{S}}$ corrected for this potential bias. ${ }^{18}$ For simplicity, subjects wearing either spectacles or contact lenses were recorded as spectacle wearers.

Siblings reported as wearing a refractive correction for myopia were contacted and asked to provide informed consent to allow their spectacle prescription to be obtained from their optometrists. However, because the response rate from the siblings was low, we sought an additional means of determining the proportion of the myopic spectacle wearing siblings who were highly myopic. Age of onset of spectacle wear (AOSW) is known to correlate with the final degree of myopia, ${ }^{19-21}$ and hence we evaluated whether an early AOSW could serve as a suitable surrogate for high myopia. Using data on families participating in a separate high-myopia genetic linkage study, the relationship between AOSW and level of myopia was modeled for all myopic subjects in the cohort over the age of 15 years with AOSW known. This data set was considered representative of the siblings of high myopes, since it comprised only high myopes and their relatives. At the time of analysis, 284 subjects in the linkage cohort met the above criteria. These subjects had refractive errors in the range of $-0.25 \mathrm{D}$ to $-20.75 \mathrm{D}$ in the least minus meridian of the right eye (RE LMM). The data were transformed to approach multivariate normality and remove heteroscedasticity. The optimal transformations were a logarithmic transformation for the AOSW, and a Box-Cox transformation for the RE LMM, as suggested by Blackie and Harris. ${ }^{22}$ The linear regression of the transformed data is shown in Figure 1A and resulted in the following model:

$$
\operatorname{sign}(\text { RE LMM }) \times(\mid \text { RE LMM } \mid)^{1 / 2}=0.9468 \times \log _{\mathrm{e}}(\text { AOSW })-4.5371
$$

This model produced physiologically plausible results and had residuals with a mean value of 0 . An AOSW of $9.1 \pm 0.7$ years corresponds to a RE LMM (95\% confidence limits) of $-6.00 \mathrm{D}(-5.67,-6.34)$.

The sensitivity and specificity of AOSW as a predictor of high myopia was also examined (Fig. 1B). There were 328 spectacle-wearing subjects (myopes and byperopes) in the linkage cohort with a known AOSW, after excluding those who were less than 15 years of age and not yet high myopes and those who wore only a presbyopic correction. Wearing spectacles from age 9 years or younger predicted high myopia with a sensitivity of $73 \%$ and a specificity of $80 \%$. The model was used to estimate the proportion of subjects expected to be high myopes on the basis of their AOSWs, not to predict the level of myopia in individual cases. Good positive predictive rates are dependent on prevalence as well as high sensitivity and specificity. The
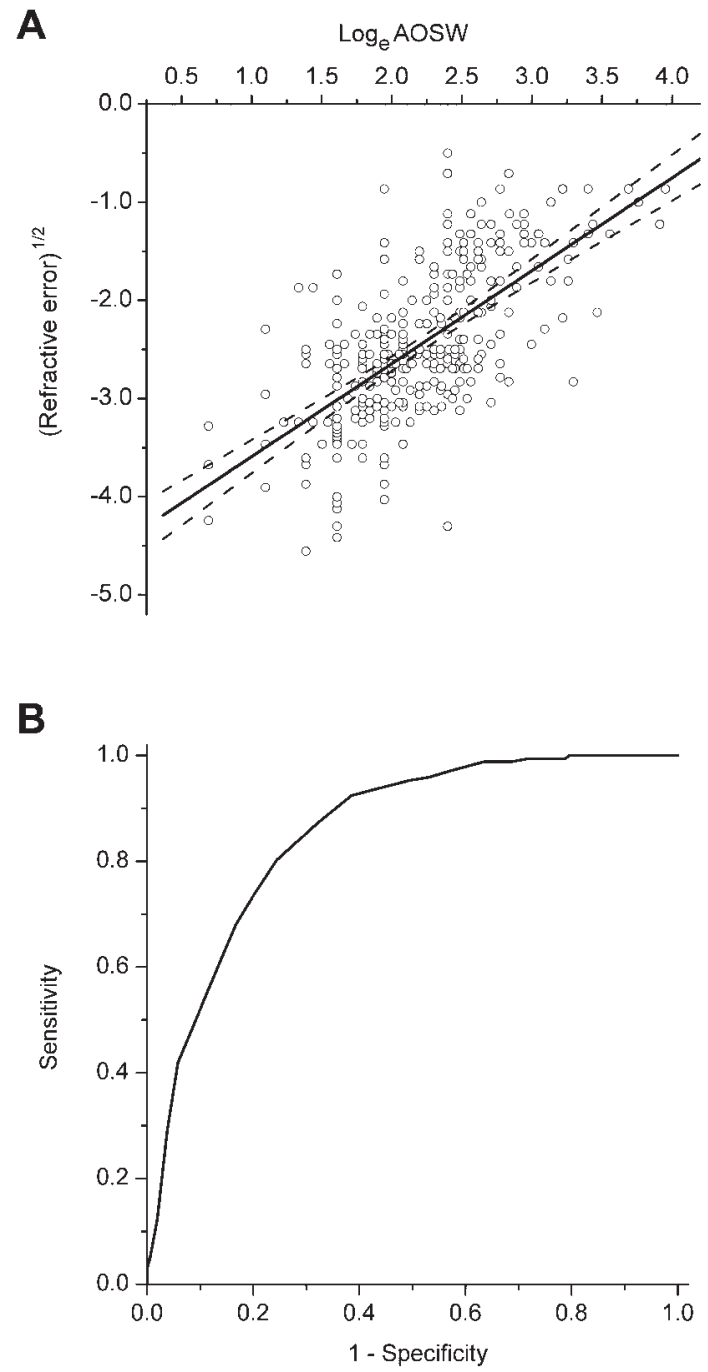

FIGURE 1. (A) The relationship between age of onset of spectacle wear (AOSW) and level of myopia. The best fit to the data (solid line; $r^{2}=0.42$ ) was for a logarithmic transformation of the AOSW and a Box-Cox type transformation of refractive error (sine $\mid \operatorname{RE}$ LMM $\left.\right|^{1 / 2}$ ). Dashed lines: show the $95 \%$ confidence limits. (B) ROC curve indicating the discrimination of high myopes from non-high myopes on the basis of AOSW (area under the curve $=0.85$, significantly different from $0.50, P<0.0001$ ). 
TABLE 2. Sibling Recurrence of Myopia and High Myopia

\begin{tabular}{lcccc}
\hline \multirow{2}{*}{$\begin{array}{l}\text { Method of } \\
\text { Contact }\end{array}$} & $\begin{array}{c}\text { Number of } \\
\text { Siblings }\end{array}$ & Myopia & High Myopia & \multirow{2}{*}{$\boldsymbol{\lambda}_{\mathbf{s}}$ High Myopia } \\
\cline { 3 - 4 } & 223 & $37.5 \%(31.0 \%-44.0 \%)$ & $7.5 \%(2.9 \%-12.9 \%)$ & $3.7 \times(1.4-6.6 \times)$ \\
\hline $\begin{array}{l}\text { Telephone } \\
\text { Mail }\end{array}$ & 270 & $50.4 \%(44.3 \%-56.5 \%)$ & $11.9 \%(6.4 \%-18.3 \%)$ & $5.9 \times(3.0-9.4 \times)$ \\
\hline
\end{tabular}

Subjects were classified as myopic if they wore spectacles full time for myopia (FTSW) and as highly myopic based on an age of onset of myopia $\leq 9.1 \pm 0.7$ year. Population prevalence $\mathrm{K}$ was taken as $2.03 \%$ $\pm 0.09 \%$ (calculated for the optometric practice population) for the calculation of $\lambda_{\mathrm{S}}$ according to the formula $\lambda_{\mathrm{S}}=\mathrm{K}_{\mathrm{S}} / \mathrm{K}$. Ninety-five percent confidence limits are shown in parentheses. For $\lambda_{\mathrm{S}}$ and $\mathrm{K}_{\mathrm{S}}$, these incorporate standard errors for both the AOSW regression model and the proportion estimates.

prevalence of high myopia among the our highly myopic probands siblings who wore spectacles for myopia full time was expected to be high enough to prevent large inaccuracies in the proportion estimates due to an excess of false positives.

To estimate the number of siblings with high myopia (those with an AOSW $\leq 9.1$ years), the cumulative frequency of siblings wearing spectacles full time for myopia (ordinate) was plotted against AOSW (abscissa). Separate plots were produced for data from subjects contacted by mail and telephone. To allow noninteger values to be modeled, the graphs were fitted with sigmoidal (Boltzmann) curves on computer (Origin 7 software; OriginLab Corp., Northampton, MA). Families $(n=7)$ containing one or more siblings younger than 15 years of age and currently unaffected were excluded from the analysis. The sibling recurrence risk $\left(K_{\mathrm{S}}\right)$, which is the proportion of siblings of affected probands who were also affected, was estimated from

$$
K_{\mathrm{s}}=\frac{\sum_{s=1}^{\infty} \sum_{a=1}^{\mathrm{s}}(a-1) n_{\mathrm{sa}}}{\sum_{s=1}^{\infty} \sum_{a=1}^{\mathrm{s}}(s-1) n_{\mathrm{sa}}}
$$

for $n_{\text {sa }}$ families with $s$ offspring, $a$ of whom are affected, which produces an unbiased estimate for single ascertainment strategies. ${ }^{18} \mathrm{~A}$ correction was made to the denominator to account for cases missing from the numerator due to an unknown age of onset, under the assumption that the proportion of high myopes among the subjects in whom this parameter was unknown would be the same as among those in whom it was known.

\section{Results}

The 161 (44.6\%) probands who replied to the postal questionnaire had a mean age of 43 years (range, 7-85 years) and a mean RE LMM of $-8.23 \mathrm{D}(-6.00$ to $-17.00 \mathrm{D})$, and $74 \%$ were female. The $135(81.3 \%)$ telephone respondents had a mean age of 45 years (range, $14-100$ ) and a mean RE LMM of $-7.86 \mathrm{D}$ $(-6.00$ to $-16.00 \mathrm{D})$, and $67 \%$ were female. Respondents to the postal and telephone questionnaires did not differ significantly in these respects from each other or from nonrespondents. None of the subjects was excluded on the basis of their responses to questions about ocular or systemic medical history. Of the 296 respondents, 8 were diabetic, 10 had cataracts, 4 had a prior history of retinal detachment or prophylactic retinal laser treatment, 27 reported a preterm birth history (this was reported to exceed 4 weeks in only 9 subjects), 5 had a history of strabismus or amblyopia, and 1 had ocular-cutaneous albinism. The number of siblings identified by the postal and telephone respondents is shown in Table 1, along with their spectacle-wearing status.

There were 16 families in which spectacle prescriptions could be obtained for some or all the siblings. In these families, there were a total of 32 siblings, 24 of whom wore a full-time spectacle correction for myopia (19 of these 24 subjects provided consent for their optometrist to be contacted). Five of the 32 siblings were affected (LMM in each eye $\leq-6.00 \mathrm{D}), 21$ were unaffected, and 6 had unknown status. The sibling recurrence risk $K_{\mathrm{S}}(95 \%$ confidence limits) in these selected families calculated on the basis of the spectacle prescription data was $19.7 \%(6.0,33.5)$. The proband-reported AOSW was known for 18 of the myopic spectacle-wearing siblings and from these data, 5 were expected to be high myopes, producing an estimate for the sibling recurrence risk of $20.3 \%(3.3,40.6)$. Thus, in this small sample the estimate from the AOSW model showed good agreement with that based on spectacle prescription data.

The prevalence of the myopia surrogate (full-time spectacle wear for myopia, FTSW) and the high myopia surrogate (AOSW $\leq 9.1$ years) in siblings of the highly myopic probands are shown in Table 2 . There was a response bias toward greater familial aggregation of myopia in postal respondents compared with the telephone respondents, which was significant for the measure of myopia (comparison of group proportions with continuity correction $z_{\mathrm{c}}=2.72, P=0.007$ ) but not for high myopia $\left(z_{\mathrm{c}}=1.37, P=0.17\right)$. As no significant difference was found between responses to the two types of questionnaire for high myopia, estimates of the sibling recurrence risk and risk ratio for the high myopia surrogate were calculated across all the data. This yielded a $K_{\mathrm{S}}$ of $10.0 \%(5.9,14.8)$ and a $\lambda_{\mathrm{S}}$ of $4.9 \%$ $(2.7,7.6)$.

Neither the recurrence of myopia (FTSW; $z_{\mathrm{c}}=0.07, P=$ 0.94 ) or high myopia (AOSW $\leq 9.1$ years; $z_{\mathrm{c}}=0.56, P=0.58$ ) was influenced by the gender of the siblings.

Because data were available from questionnaires on the FTSW status of parents, it was possible to investigate whether parental myopia had an influence on the recurrence of myopia in the siblings of the highly myopic probands (Fig. 2). Cases in which neither parent wore spectacles for myopia were surprisingly common (Table 3), accounting for $39.6 \%$ of high myopes. The siblings of probands without myopic parents showed a lower than average recurrence of FTSW, 26.0\% (19.8,32.2), and our high-myopia surrogate, $5.6 \%(1.8,9.8)$. The recurrence of high myopia was increased in siblings of high myopes who had one parent who was a full-time myopic spectacle wearer, $11.2 \%(4.8,18.8)$, but this increase was not significant $\left(z_{\mathrm{c}}=\right.$ $1.76, P=0.08$ ) and also was not influenced by whether it was their mother or father who wore a myopic correction $\left(z_{\mathrm{c}}=\right.$ $0.05, P=0.96)$. There was a further increase, which again did not reach statistical significance $\left(z_{\mathrm{c}}=1.80, P=0.07\right)$, if both parents were myopic spectacle wearers, $21.8 \%(9.3,35.3)$. 


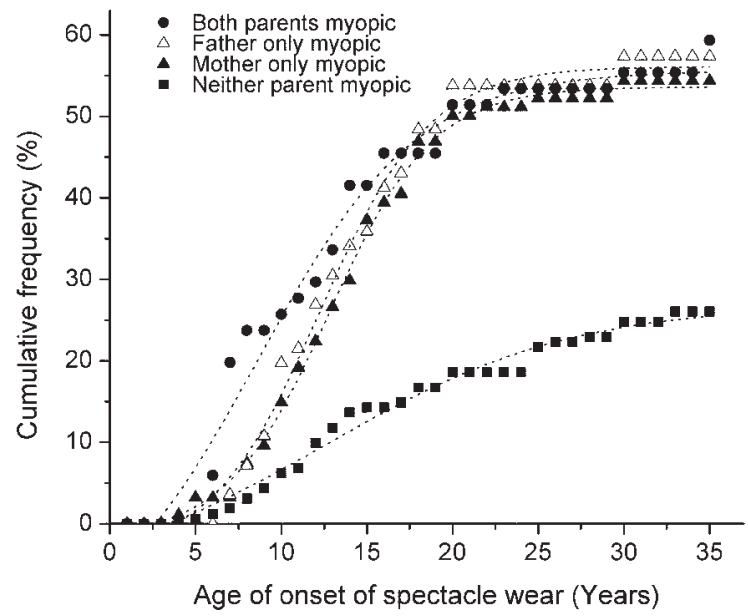

Figure 2. Proportion of siblings wearing spectacles full time for myopia, as a function of the number of myopic parents and the AOSW.

However, probands with at least one myopic parent were more likely to have highly myopic siblings than those with no myopic parents $\left(z_{\mathrm{c}}=2.67, P=0.007\right)$.

\section{Discussion}

The sibling recurrence risk for high myopia estimated on the basis of an AOSW $\leq 9.1$ years $\left(K_{\mathrm{S}} \sim 10.0 \%\right)$ is similar to those found in the only two previous studies, despite differences in ascertainment strategy, data collection method, and refractive criteria. Guggenheim et al. ${ }^{23}$ reanalyzed a young (age, 13-14 years) cohort studied by Goldschmidt ${ }^{24}$ and found a $K_{\mathrm{S}}$ of $\sim 9.1 \%$. The low prevalence $(0.45 \%)$ of high myopia (mean spherical refractive error in either eye $<-6.00 \mathrm{D}$ ) in Goldschmidt's cohort meant that, despite a large initial sample size, the estimate of $K_{\mathrm{S}}$ was based on data from only 31 families. The exclusion of families with young siblings in the present study may have contributed to the slightly higher $K_{\mathrm{s}}$ estimate, compared with that calculated from Goldschmidt's data. The estimate by Tanabe and Fujiki ${ }^{25}$ of $K_{\mathrm{S}}$ for higher myopia ( $\leq$ $-8.00 \mathrm{D}$ ) in a Japanese cohort was $12.3 \%$. Although they used a higher threshold (more extreme proband selection) than we used, this may have been balanced by the higher prevalence of myopia in East Asian populations. ${ }^{26,27}$

The estimate of $\lambda_{\mathrm{s}}(95 \%$ confidence limits) from this study is $4.9(2.7,7.6)$, considerably lower than the only previous estimate of $19.8(1.0,54.6) .^{23}$ Although the approaches taken in reaching these estimates both have their limitations, these factors were at least largely independent in the two studies. In the present investigation, the population prevalence of high myopia is most likely an overestimate due to the clinical selec- tion of the cohort. This bias will make the estimate of $\lambda_{\mathrm{s}}$ overly conservative. The converse is probably true in relation to the cohort studied by Goldschmidt, ${ }^{24}$ due to the young age of his cohort. If the assumption of single ascertainment is incorrect, it would also serve to reduce the estimate of $\lambda_{\mathrm{s}}$.

An indirect approach to the estimation of the sibling recurrence was used in this study to maximize the proportion of families on which the estimate could be based, in an attempt to avoid response bias in favor of probands with a positive family history. Hence, the proportion of myopic siblings who were high myopes was estimated using an age of onset of spectacle wear for myopia of 9.1 years or younger as reported by the proband. The relationship of age of onset of spectacle wear and the degree of myopia has been established in previous studies and was demonstrated here in both regression and receiver operating characteristic (ROC) models. In addition this indirect approach showed good agreement with actual recurrence values in the small number of families in which the siblings' refractive error was known. The potential for overreporting of sibling affectation by affected probands has been described by Guo. ${ }^{16}$ However, this does not appear to have occurred in the present study, as the high myopia recurrence estimate in siblings in whom the affectation status could be confirmed was no lower than the overall estimate. A comparison of the estimates for the postal and telephone questionnaires indicated that response bias in favor of probands with highly myopic siblings was also not a major problem, although this type of response bias did appear to occur for low myopia to a small extent.

In the present study, highly myopic probands with no myopic parents were common (39.6\%). This is surprising in light of the models developed by Yap et al. ${ }^{28}$ and Hirsch and Ditmars, ${ }^{29}$ which predicted that individuals with high myopia would have at least one myopic parent. Such a finding is, however, consistent with the cohort studied by Goldschmidt, ${ }^{24}$ in which neither parent was myopic (spherical equivalent $\leq-0.50 \mathrm{D}$ in each eye) in $59 \%$ of cases.

Probands in the present study with no myopic parents were much less likely to have highly myopic siblings $\left(K_{\mathrm{S}} \sim 5.6 \%\right)$, but this recurrence still exceeded the likely maximum population prevalence of $2.03 \%(z=3.22, P=0.001)$. This implies that, in at least some of these families, genetic and/or shared family environment risk factors contribute to the likelihood of development of high myopia, rather than unique environment effects alone. Having at least one myopic parent increases the risk to the siblings of highly myopic probands. However, the fact that the parents of high myopes seem to be no more likely to be myopic than do those of low myopes, ${ }^{26,28,30}$ indicates that high myopia cannot be viewed merely as a higher threshold on an additive polygenic risk model for refractive error.

As sibling recurrence risk is equivalent to a weighted average segregation ratio over all mating types, ${ }^{18}$ our $K_{\mathrm{S}}$ estimate of $\sim 10 \%$ for high myopia is well below the $\sim 50 \%$ level that would be expected if all cases were caused by the rare, highly

TABLE 3. Proportions of Parents who Wore Spectacles Full Time for Myopia

\begin{tabular}{lllr}
\hline & $\begin{array}{c}\text { Telephone } \\
(\boldsymbol{n}=\mathbf{1 3 2})\end{array}$ & $\begin{array}{c}\text { Mail } \\
(\boldsymbol{n}=\mathbf{1 5 7})\end{array}$ & \multicolumn{1}{c}{ Total } \\
\hline Father only myopic & $19 / 123(15.4 \%)$ & $27 / 153(17.6 \%)$ & $46(16.7 \%)$ \\
Mother only myopic & $30 / 124(24.2 \%)$ & $34 / 153(22.2 \%)$ & $64(23.1 \%)$ \\
Both parents myopic & $16 / 125(12.8 \%)$ & $20 / 153(13.1 \%)$ & $36(12.9 \%)$ \\
Neither parent myopic & $49 / 122(40.2 \%)$ & $60 / 153(39.2 \%)$ & $109(39.6 \%)$ \\
\hline
\end{tabular}

Parents for whom spectacle wearing status was unknown, or who wore spectacles part time were omitted from the analysis. 
penetrant dominant loci for which linkage analysis has been successful. ${ }^{6-8}$ In addition, if the recurrence in siblings were solely under the control of additive polygenes (i.e., assuming a normally distributed trait with a heritability of 1.0), the $\lambda_{\mathrm{s}}$ would still be on the order of $\sim 10$ for a condition with a prevalence of $2 \%{ }^{31}$ As recent twin studies have attributed most of the variance in refractive error to additive genetic effects $^{32,33}$ and linkage studies have shown that high myopia can result from allelic variants with large effects, it would seem that high myopia represents both the tail of the distribution of these additive genetic effects and a number of distinct etiological "outliers" under the control of rare genes of large effect and environmental events. ${ }^{34}$

Our data suggest that approximately 1 in 10 high myopes in the United Kingdom has a highly myopic sibling. Probands with myopic parents are more likely to have highly myopic siblings; and, as a consequence, recruitment strategies such as the affected sib pair study design would be much more likely to ascertain families in which the parents are also myopic. Such selective ascertainment schemes would therefore tend to overemphasize the importance of dominant genes. ${ }^{35,36}$ Certainly, the frequent occurrence of high myopes without myopic parents in this study indicates that recessive genes may well contribute significantly to the risk of high myopia. The $\lambda_{\mathrm{s}}$ estimate of $\sim 5$ is comparable with that of other complex traits in which susceptibility loci have been successfully mapped (for example, Refs. 37, 38). However, the power of a linkage study to detect a particular locus is determined by the specific genotype's relative risk, which is unlikely to be similar for each contributory locus and does not exhibit a simple relationship to $\lambda_{\mathrm{s}}{ }^{39}$ Our data indicate that high myopia is rarely caused by noninteracting, large-effect, high-penetrance, dominant loci. Thus, large study cohorts, or those composed of large pedigrees, may well be necessary to map the common contributory loci of high myopia.

\section{Acknowledgments}

The authors thank Rowanne Black, Helen Fletcher, and Emma Farrow for assistance during summer student placements supported by grants from the Wellcome Trust and Nuffield Foundation, and the participating families and the staff and patients of Conlons Opticians.

\section{References}

1. Working Group on Myopia Prevalence and Progression NRC. Myopia: Prevalence and Progression. Washington DC: National Academy Press; 1989.

2. Katz J, Tielsch JM, Sommer A. Prevalence and risk factors for refractive errors in an adult inner city population. Invest Ophthalmol Visual Sci. 1997;38:334-340.

3. Tron EJ. The optical elements of the refractive power of the eye. In: Ridley F, Sorsby A, eds. Modern Trends in Ophthalmology. New York: Paul B. Hoeber; 1940.

4. Hirsch MJ. Analysis of inhomogeneity of myopia in adults. Am J Optom. 1950;27:562-571.

5. Sorsby A, Benjamin B. Modes of inheritance of errors of refraction. J Med Genet. 1973;10:161-164.

6. Young TL, Ronan SM, Drahozal LA, et al. Evidence that a locus for familial high myopia maps to chromosome 18p. Am J Hum Genet. 1998;63:109-119.

7. Young TL, Ronan SM, Alvear AB, et al. A second locus for familial high myopia maps to chromosome 12q. Am J Hum Genet. 1998; 63:1419-1424.

8. Paluru P, Ronan SM, Heon E, et al. New locus for autosomal dominant high myopia maps to the long arm of chromosome 17. Invest Ophthalmol Vis Sci. 2003;44:1830-1836.
9. Naiglin L, Gazagne C, Dallongeville F, et al. A genome wide scan for familial high myopia suggests a novel locus on chromosome 7q36. J Med Genet. 2002;39:118-124.

10. Durner M, Greenberg DA, Hodge SE. Inter- and intrafamilial heterogeneity: effective sampling strategies and comparison of analysis methods. Am J Hum Genet. 1992;51:859-870.

11. Risch N. Assessing the role of HLA-linked and unlinked determinants of disease. Am J Hum Genet. 1987;40:1-14.

12. Risch N. Linkage strategies for genetically complex traits. II. The power of affected relative pairs. Am J Hum Genet. 1990;46:229241.

13. Schliekelman $P$, Slatkin $M$. Multiplex relative risk and estimation of the number of loci underlying an inherited disease. Am J Hum Genet. 2002;71:1369-1385.

14. Guo S-W. Sibling recurrence risk ratio as a measure of genetic effect: caveat emptor! Am J Hum Genet. 2002;70:818-819.

15. Guo S-W. Familial aggregation of environmental risk factors and familial aggregation of disease. Am J Epidemiol. 2000;151:11211131.

16. Guo S-W. Inflation of sibling recurrence-risk ratio, due to ascertainment bias and/or overreporting. Am J Hum Genet. 1998;63: 252-258.

17. Walline JJ, Zadnik K, Mutti DO. Validity of surveys reporting myopia, astigmatism, and presbyopia. Optom Vis Sci. 1996;73: 376-381.

18. Olson JM, Cordell HJ. Ascertainment bias in the estimation of sibling genetic risk parameters. Genet Epidemiol. 2000;18:217235.

19. Goss DA. Variables related to the rate of childhood myopia progression. Optom Vis Sci. 1990;67:631-636.

20. Chew SJ, Ritch R, Leong YK, et al. The age of onset of myopia is a predictor of adult myopia severity. In: Shimizu K, ed. Current Aspects in Ophthalmology: Proceedings of the XIII Congress of the Asia Pacific Academy of Ophthalmology. Amsterdam: Elsevier; 1992:680-685.

21. Parssinen O, Lyyra A. Myopia and myopic progression among schoolchildren: a three year follow up study. Invest Ophthalmol Vis Sci. 1993;34:2794-2802.

22. Blackie CA, Harris WF. Refraction and keratometry: departures from and transformations toward multivariate normality. Optom Vis Sci. 1997;74:452-458.

23. Guggenheim JA, Kirov G, Hodson SA. The heritability of high myopia: a re-analysis of Goldschmidt's data. J Med Genet. 2000; 37:227-231.

24. Goldschmidt E. On the etiology of myopia. Acta Ophthalmol. 1968;(Suppl) 98.

25. Tanabe U, Fujiki K. Genetic analysis of myopia [in Japanese]. Folia Ophthalmol Jpn. 1989;40:776-784.

26. Ashton GC. Segregation analysis of ocular refraction and myopia. Hum Hered. 1985;35:232-239.

27. Lin LL-K, Shih Y-F, Hsiao CK, et al. Epidemiologic study of the prevalence and severity of myopia among schoolchildren in Taiwan in 2000. J Formos Med Assoc. 2001;100:684-691.

28. Yap M, Wu M, Liu ZM, Lee FL, Wang SH. Role of heredity in the genesis of myopia. Ophthalmic Physiol Opt. 1993;13:316-319.

29. Hirsch MJ, Ditmars DL. Refraction of young myopes and their parents: a re-analysis. Am J Optom. 1969;46:30-32.

30. Wu MM, Edwards MH. The effect of having myopic parents: an analysis of myopia in three generations. Optom Vis Sci. 1999;76: 387-392.

31. Cavalli-Sforza LL, Bodmer WF. The Genetics of Human Populations. San Francisco: WH Freeman; 1971.

32. Hammond CJ, Snieder H, Gilbert CE, Spector TD. Genes and environment in refractive error: the twin eye study. Invest Ophthalmol Vis Sci. 2001;42:1232-1236.

33. Lyhne N, Sjølie AK, Kyvik KO, Green A. The importance of genes and environment for ocular refraction and its determiners: a population based study among 20-45 year old twins. Br J Ophthalmol. 2001;85:1470-1476.

34. Bear JC. Epidemiology and genetics of refractive anomalies. In: Grosvenor T, Flom MC, eds. Refractive Anomalies: Research and 
Clinical Applications. Boston: Butterworth Heinemann; 1991:5781.

35. Delbono EA, Gwiazda JE, Haines JL, Wiggs JL. Analysis of pedigrees affected by juvenile onset myopia: evidence for autosomal dominant inheritance (abstract). Am J Hum Genet. 1995; 57:87.

36. Naiglin L, Clayton J, Gazagne C, et al. Familial high myopia: evidence of an autosomal dominant mode of inheritance and genetic heterogeneity. Ann Genet. 1999;42:140-146.
37. Puca AA, Daly MJ, Brewster SJ, et al. A genome-wide scan for linkage to human exceptional longevity identifies a locus on chromosome 4. Proc Natl Acad Sci USA. 2001;98:10505-10508.

38. Myers A, Holmans $\mathrm{P}$, Marshall $\mathrm{H}$, et al. Susceptibility locus for Alzheimer's disease on chromosome 10. Science. 2000;290:23042305.

39. Rybicki BA, Elston RC. The relationship between the sibling recurrence-risk ratio and genotype relative risk. Am J Hum Genet. 2000;66:593-604.

\section{$\begin{array}{lllllll}\mathbf{E} & \mathbf{R} & \mathbf{R} & \mathbf{A} & \mathbf{T} & \mathbf{U} & \mathbf{M}\end{array}$}

Erratum in: "Efficacy and Safety of Memantine Treatment for Reduction of Changes Associated with Experimental Glaucoma in Monkey, I: Functional Measures" by Hare et al. (Invest Ophthalmol Vis Sci. 2004;45:2625-2639).

The DOI for this article was misprinted in the August issue. The correct DOI is 10.1167/ iovs.03-0566. 